\title{
Consumo alimentar de crianças com base na pirâmide alimentar brasileira infantil
}

\author{
Food intake by children based on the Brazilian \\ food guide pyramid for young children
}

Roseane Moreira Sampaio BARBOSA ${ }^{1,2}$

Carolina $\mathrm{CROCCIA}^{3}$

Carolina Guimarães do Nascimento CARVALHO ${ }^{3}$

Viviane Carvalho FRANCO $^{3}$

Rosana SALLES-COSTA ${ }^{4}$

Eliane Abreu SOARES ${ }^{4,5}$

\section{R E S U M O}

\section{Objetivo}

Este estudo tem como objetivo comparar o consumo alimentar durante a semana com o do final de semana de vinte crianças de uma creche filantrópica, com base na pirâmide alimentar de crianças brasileiras de dois e três anos, proposta por Philippi.

\section{Métodos}

A avaliação sociodemográfica foi realizada por meio das variáveis: renda mensal familiar, escolaridade, condições de moradia e saneamento básico. Para a avaliação dietética foram utilizados os métodos de pesagem direta de alimentos (na creche) e registro alimentar (na residência) após três meses de freqüência da criança na creche. Os alimentos e preparações consumidos pelas crianças durante a semana e no final de semana foram transformados em porções em função dos oito grupos de alimentos correspondentes, conforme recomendado por Philippi. Posteriormente, compararam-se as médias das porções consumidas no final de semana com o consumo semanal de cada grupo de alimentos, utilizando o teste " $t$ " de Student para verificar significância estatística entre os grupos, considerando o nível de significância de $5 \%$.

\footnotetext{
1 Mestranda, Programa de Pós-Graduação em Nutrição Humana, Instituto de Nutrição, Centro de Ciências da Saúde, Universidade Federal do Rio de Janeiro. Av. Pedro II, 260, 22410-002, Rio de Janeiro, RJ, Brasil. Correspondência para/Correspondence to: R.M.S. BARBOSA. E-mail: <roseanesampaio@ig.com.br>.

2 Fundação Ataulpho de Paiva. Rio de Janeiro, RJ, Brasil.

3 Acadêmicas, Instituto de Nutrição, Centro de Ciências da Saúde, Universiade Federal do Rio de Janeiro. Rio de Janeiro, RJ, Brasil.

${ }^{4}$ Instituto de Nutrição, Centro de Ciências da Saúde, Universidade Federal do Rio de Janeiro. Rio de janeiro, RJ, Brasil.

5 Instituto de Nutrição, Universidade do Estado do Rio de Janeiro. Rio de janeiro, RJ, Brasil.
} 


\section{Resultados}

Observou-se uma diferença significativa $(p<0,05)$ entre a média de porções de carnes, leguminosas, legumes e frutas consumidos nos dois momentos avaliados.

\section{Conclusão}

A dieta do final de semana é mais inadequada do que a dieta oferecida às crianças durante a semana.

Termo de indexação: consumo alimentar, criança, pirâmide alimentar, nutrição da criança.

\section{A B S T R A C T}

\section{Objective}

The objective of this study was to compare the food consumption during the week of twenty children at a nonprofit day care center, with that consumed at the weekend, based on the Brazilian Food Guide Pyramid for two to three year old children proposed by Philippi.

\section{Methods}

The social demographic evaluation was based on the following variables: income, educational level, housing and sanitation conditions. The food intake was measured by direct weighing of the food (in the day care center) and registry of the food consumed (at home), after three months attendance of the child at the day care center. The food and meals consumed by the children during the week and at the weekend were transformed into portions based on the eight corresponding food groups, according to Philippi. The average daily consumption of each food group, in portions, at the weekend, was then compared with that consumed on weekdays using the Student " $t$ " test, considering a 5\% significance level.

\section{Results}

A significant difference $(\mathrm{p}<0.05)$ was observed between the average number of portions of meat, legumes, vegetables and fruits consumed in the two periods evaluated.

\section{Conclusion}

The results suggest inadequacy of the diet consumed by the children at the weekend when compared to that consumed during the week.

Indexing Terms: food consumption, child, food guide pyramid, child nutrition.

\section{N T R O D U Ç Ã O}

Os guias alimentares têm sido utilizados para descrever as recomendações quantitativas e qualitativas dos padrões dietéticos baseados em evidências científicas' ${ }^{1}$. De acordo com a Food and Agriculture Organization/World Health Organization", "os guias alimentares oferecem recomendações dietéticas através de comunicados à população para promover o bem-estar nutricional".

O primeiro guia alimentar foi proposto por Caroline Hunt, no ano de 1916, e traduz as recomendações de uma dieta saudável. Por volta de 1940, após as novas recomendações do NRC de 1941, foi desenvolvido um guia alimentar popularmente chamado Basic 7, que tinha a intenção de orientar as pessoas a se alimentarem sozinhas, porém era muito complexo e ainda faltavam as porções dos alimentos. Em 1956, especialistas em nutrição publicaram um novo guia alimentar, o Basic 4, com números de porções recomendadas dos quatro grupos de alimentos: leite, carnes, vegetais e frutas e pães e cereais ${ }^{3}$.

A partir da década de 1970, pesquisadores associaram o alto consumo de certos alimentos com o aparecimento de determinadas doenças ${ }^{3}$. Foram elaborados diversos guias alimentares e, em 1979, pela primeira vez, foi acrescentado o quinto grupo com alimentos que forneciam energia a partir de gorduras e açúcares de adição 
e álcool, chamando a atenção para a necessidade do seu consumo moderado ${ }^{3}$.

Nos anos 1980 e 1990 enfatizaram-se não somente as quantidades recomendadas dos diferentes grupos de alimentos, mas também o aspecto qualitativo baseado na sua composição em nutrientes ${ }^{4}$.

O mais recente guia alimentar, a pirâmide alimentar, foi desenvolvido pelo Departamento de Agricultura dos Estados Unidos (USDA) em 19925 sendo representado graficamente por uma pirâmide e tendo como princípio a variedade, moderação e proporcionalidade. Amplamente reconhecida como uma ferramenta de educação nutricional, a pirâmide é utilizada para monitorar o consumo atual e recomendado dos cinco maiores grupos de alimentos ${ }^{6}$. A forma gráfica de distribuição de alimentos permite uma fácil compreensão por parte da população, fazendo com que haja o consumo de vários alimentos e em quantidade suficiente para juntos comporem uma dieta adequada nutricionalmente ${ }^{7}$.

Em 1999, o USDA realizou uma adaptação da pirâmide alimentar para crianças de dois a seis anos de idade com o objetivo de focar as preferências alimentares e as recomendações nutricionais para essa faixa etária ${ }^{8}$. Sabe-se que, nessa fase, a criança deve consumir a quantidade de alimentos necessária para alcançar seu potencial de crescimento. O atraso no crescimento devido às circunstâncias nutricionais e ambientais está associado com maiores taxas de morbimortalidade, com dificuldades no aprendizado e com menor capacidade física e intelectual na vida adulta ${ }^{9}$

Nessa faixa etária há necessidade de um maior cuidado em relação à alimentação, principalmente pelo fato de ocorrer a incorporação de novos hábitos alimentares que implica o conhecimento de novos sabores, texturas e cores, experiências sensoriais que irão influenciar diretamente o padrão alimentar a ser adotado pelo infante $^{10}$

Considerando a complexidade dos fatores envolvidos na alimentação da criança e a dificulda- de na oferta de uma dieta adequada, Philippi et al. $^{10}$ desenvolveram um instrumento para orientação nutricional com base na proposta da pirâmide alimentar Norte-Americana, adaptada às crianças brasileiras de dois e três anos de idade. Essa pirâmide foi baseada em uma dieta padrão para essa faixa etária, contendo os alimentos mais comumente consumidos ${ }^{10}$.

Ela é útil no monitoramento nutricional para verificar as diferenças entre o consumo alimentar atual e o recomendado. Vem sendo utilizada como uma ferramenta de educação nutricional, além de avaliar os padrões do consumo dietético das populações ${ }^{10}$. Este estudo tem como objetivo comparar o consumo alimentar durante a semana na instituição, complementado com o consumo na residência com a dieta do final de semana de crianças pertencentes a uma creche filantrópica.

\section{M É T O D O S}

O desenho deste estudo é transversal e os dados foram coletados pela própria pesquisadora e três estagiárias treinadas. Participaram da pesquisa todas as crianças $(n=20)$, de dois e três anos, de ambos os sexos, matriculadas no ano de 2003 em uma creche filantrópica na llha de Paquetá, Rio de Janeiro. Esta pesquisa foi aprovada pelo Comitê de Ética em Pesquisa do Instituto de Puericultura e Pediatria Martagão Gesteira da Universidade Federal do Rio de Janeiro sob o $n^{\circ} 04 / 03$.

A avaliação socioeconômica foi determinada com a aplicação de um questionário constando de informações sobre renda mensal familiar, escolaridade do responsável, condições de moradia e saneamento básico.

A avaliação dietética do dia da semana (creche + residência) foi realizada após três meses de freqüência da criança na creche. Foi analisada a composição nutricional das refeições consumidas durante dois dias não consecutivos na creche (desjejum, colação, almoço, merenda e jantar) 
utilizando o método de pesagem direta de alimentos ${ }^{11}$.

Para quantificar os alimentos consumidos na creche, os alimentos sólidos foram pesados com auxílio de uma balança digital da marca Plena com capacidade de dois quilos, com escala de um grama, e os líquidos foram medidos com auxílio de recipientes graduados (com graduação de $10 \mathrm{~mL}$ e capacidade máxima de $250 \mathrm{~mL}$ ), sendo todos os valores registrados em formulários próprios. Ao final de cada refeição foram pesadas as sobras alimentares individuais do prato ou caneca de cada criança. Para a obtenção da sobra de cada alimento das preparações fornecidas foi considerado o peso da sobra proporcional ao peso dos alimentos dessas preparações inicialmente porcionadas na refeição. Após esse cálculo foi obtido o consumo alimentar individual de cada alimento, para cada criança, por meio da fórmula: consumo alimentar individual= porção oferecida - sobra alimentar individual.

Nesse período de dois dias, para estimar outros possíveis alimentos e bebidas consumidos pela criança em sua residência (antes e depois do período de permanência na creche), foi solicitado ao responsável o preenchimento do registro alimentar, indicando, por meio de medidas caseiras, os alimentos consumidos a fim de complementar o consumo alimentar do dia.

Para complementar a informação do consumo dietético semanal, foi realizado pelos responsáveis o registro alimentar de um dia do final de semana (FS) nas residências das crianças.

Após estimar o consumo alimentar durante a semana e no final de semana, os alimentos e preparações consumidos pelas crianças foram transformados em porções em função dos oito grupos de alimentos correspondentes (cereais, vegetais, frutas, leguminosas, carnes, leite, gordura e açúcar). Tais porções dos grupos de alimentos consumidas, tanto no final de semana quanto durante a semana, foram comparadas com a pirâmide alimentar para crianças de dois e três anos recomendada por Philippi ${ }^{10}$ para verificar possíveis inadequações.

Para comparar o consumo do final de semana com o consumo semanal de cada grupo de alimentos, compararam-se as médias das porções utilizando o teste " $\mathrm{t}$ " de Student para verificar significância estatística entre os grupos, considerando o nível de significância de 5\% $(p<0,05)$. Os procedimentos de análise foram realizados no programa STATA versão 6.0.

\section{RES U L T A D OS}

Em relação às condições sociodemográficas das crianças, verificou-se que todas as famílias residiam em casa de alvenaria com acesso ao saneamento básico (água, esgoto e coleta de lixo). Cerca de $40 \%$ das famílias eram constituídas por cinco ou mais pessoas no mesmo domicílio, $70 \%$ tinham o pai como o chefe da família e em $90 \%$ das famílias a mãe era a pessoa que tinha maior conhecimento sobre a saúde da criança. Quanto à escolaridade, observou-se que 60\% das mães e $75 \%$ dos chefes de família possuíam o ensino fundamental incompleto.

Quanto à distribuição da renda mensal familiar segundo tercis, a amplitude variou de $R \$ 200,00$ a $R \$ 2.800,00$, sendo a renda mensal familiar per capita $\mathrm{R} \$ 100,50$ ( $1^{\circ}$ tercil), $\mathrm{R} \$ 145,28$ ( $2^{\circ}$ tercil) e $\mathrm{R} \$ 277,5$ ( $3^{\circ}$ tercil) (Tabela 1 ).

Comparando o consumo durante a semana com o do final de semana observa-se uma diferença significativa $(p<0,05)$ entre a média de porções de carnes, leguminosas, frutas e legumes $(p<0,001)$. O consumo do grupo das carnes no final de semana foi menor do que a porção consumida durante a semana. O consumo de leguminosas durante a semana foi maior do que no final de semana. No grupo das frutas e legumes, a porção consumida no final de semana foi menor do que a recomendada pela pirâmide alimentar brasileira, principalmente em relação à porção de legumes. Apesar de o grupo das gorduras e do leite ser menos consumido no final 
Tabela 1. Distribuição das características sociodemográficas de crianças matriculadas em uma creche filantrópica na llha de Paquetá, Rio de Janeiro.

\begin{tabular}{|c|c|c|}
\hline \multirow{2}{*}{ Características } & \multicolumn{2}{|c|}{ Crianças } \\
\hline & $\mathrm{n}$ & $\%$ \\
\hline \multicolumn{3}{|l|}{ Tipo de construção } \\
\hline Alvenaria & 20 & 100 \\
\hline \multicolumn{3}{|l|}{ Abastecimento de água } \\
\hline Rede pública com canalização & 18 & 85 \\
\hline Rede pública e poço & 2 & 15 \\
\hline \multicolumn{3}{|l|}{ Rede de esgoto } \\
\hline Rede pública & 20 & 100 \\
\hline \multicolumn{3}{|l|}{ Coleta de lixo } \\
\hline Regularmente & 20 & 100 \\
\hline \multicolumn{3}{|l|}{ Número de pessoas no domicílio } \\
\hline 2 & 1 & 5 \\
\hline 3 & 7 & 35 \\
\hline 4 & 4 & 20 \\
\hline$\geq 5$ & 8 & 40 \\
\hline \multicolumn{3}{|l|}{ Chefe da Família } \\
\hline Pai & 14 & 70 \\
\hline Mãe & 1 & 10 \\
\hline Avó & 1 & 5 \\
\hline Outros & 4 & 15 \\
\hline \multicolumn{3}{|l|}{ Escolaridade do chefe } \\
\hline Fundamental incompleto & 15 & 75 \\
\hline Fundamental $2^{\circ}$ grau incompleto & 2 & 10 \\
\hline Médio & 2 & 10 \\
\hline Universitário & 1 & 5 \\
\hline \multicolumn{3}{|c|}{ Pessoa que conhece a saúde da criança } \\
\hline Mãe & 18 & 90 \\
\hline Tia & 1 & 5 \\
\hline \multicolumn{3}{|l|}{ Escolaridade da mãe } \\
\hline Fundamental incompleto & 12 & 60 \\
\hline Fundamental $2^{\circ}$ grau incompleto & 3 & 15 \\
\hline Médio & 4 & 20 \\
\hline Superior completo & 1 & 5 \\
\hline \multicolumn{3}{|l|}{ Tercis de renda mensal familiar } \\
\hline $1^{\circ}$ tercil - até $R \$ 500,00$ & 6 & 30 \\
\hline $2^{\circ}$ tercil - $\mathrm{R} \$ 501,00$ a $>\mathrm{R} \$ 800,00$ & 7 & 35 \\
\hline $3^{\circ}$ tercil $-\mathrm{R} \$>800,00$ & 7 & 35 \\
\hline
\end{tabular}

de semana e de o grupo dos cereais ser mais consumido nesse período, as diferenças encontradas não foram significativas.

Quanto à comparação entre as porções consumidas e as porções recomendadas pela pirâmide alimentar brasileira infantillio, observou-se que durante a semana a média do consumo de açúcar e de feijão foi três vezes maior do que

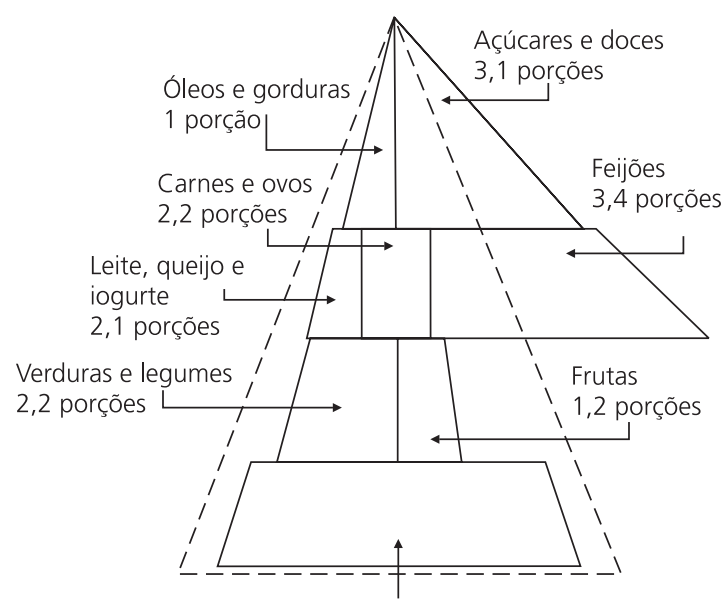

Arroz, pão, massa, batata, mandioca - 4,2 porções

Figura 1. Pirâmide alimentar infantil durante a semana.

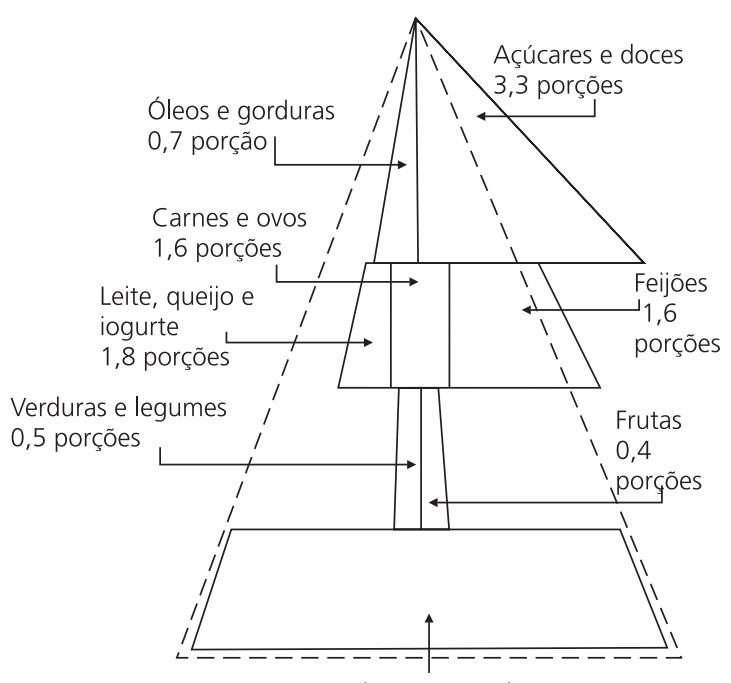

Arroz, pão, massa, batata, mandioca $-4,8$ porções

Figura 2. Pirâmide alimentar infantil do final de semana.

o recomendado (Figura 1). A média do consumo de carne e gordura foi adequada e a média dos demais grupos alimentares (legumes, frutas, leite) foi abaixo da recomendada. No final de semana (Figura 2) o consumo de todos os grupos, com exceção do grupo do açúcar e das leguminosas, foi abaixo das porções recomendadas por Philippi $^{10}$. 


\section{DISCUSSÃO}

As práticas de alimentação são importantes determinantes das condições de saúde na infância e estão fortemente condicionadas ao poder aquisitivo das famílias, do qual dependem a disponibilidade, a quantidade e a qualidade dos alimentos consumidos ${ }^{12}$.

A avaliação do consumo alimentar em pesquisas destinadas a estabelecer condições de saúde torna-se necessária, pois permite caracterizar o nível de risco e a vulnerabilidade da população às deficiências nutricionais, assim como adequar ou propor medidas de intervenção que garantam a saúde, particularmente no segmento da população menor de cinco anos, idade na qual a dieta constitui um dos fatores determinantes da velocidade de crescimento e desenvolvimento ${ }^{13}$.

Para avaliar o consumo alimentar deve-se levar em consideração a variabilidade intra e entre indivíduos, pois essa variação é um dos fatores que reduzem a precisão do método para estimar o consumo alimentar. Erros podem ocorrer em estudos dietéticos que avaliam somente um dia do consumo alimentar, pois os indivíduos não consomem os mesmos alimentos todos os dias ${ }^{14}$. A coleta do consumo alimentar das crianças foi realizada durante três dias (dois dias da semana e um dia de final de semana) e, de acordo com alguns autores, esse período é suficiente para obter o consumo alimentar médio de um grupo de indivíduos ${ }^{15,16}$. Utilizou-se o método de pesagem de alimentos para avaliar o consumo alimentar, pois, segundo Menchú ${ }^{17}$, é um dos métodos mais precisos para estimar o consumo usual.

No presente estudo (Tabela 2), observou-se que o consumo de açúcar foi três vezes maior que a porção recomendada (uma porção) por Phillippi et al. ${ }^{10}$, tanto durante a semana como no final de semana, principalmente pelo alto consumo de refrescos industrializados, refrigerantes, balas e açúcar de adição. Sabe-se que esses refrescos industrializados não oferecem a variedade de nutrientes encontrados em sucos naturais, além de serem adicionados de açúcar ${ }^{18}$. No estudo nacional, realizado em 1994, com 1810 crianças de dois a dezoito anos encontrou-se um consumo médio de bebidas industrializadas de 150ml por dia ${ }^{19}$. Aquino \& Philippi ${ }^{12}$, estudando o consumo infantil de alimentos industrializados, encontraram um alto consumo de açúcar em crianças com menor poder aquisitivo ( $1^{\circ}$ quartil renda per capita $R \$ 59,19)$ e, de acordo com a Associação Brasileira das Indústrias de Alimentação, a produção de refrigerantes vem apontando significativo aumento nos últimos anos $^{20,21}$. Nesta pesquisa encontrou-se um alto consumo de açúcar em todos os tercis de renda. Conforme dados do "Estudo multicêntrico sobre consumo alimentar", realizado nas cidades de Campinas e Goiânia, os refrigerantes foram consumidos em todas as faixas de renda e a participação no valor energético total da dieta diminuiu conforme o aumento da renda da família ${ }^{21}$. Resultado semelhante foi encontrado por Sichieri22, no estudo realizado em uma amostra

Tabela 2. Comparação da média do número de porções consumidas dos diferentes grupos de alimentos pelas crianças matriculadas em uma creche filantrópica durante a semana e no final de semana.

\begin{tabular}{|c|c|c|c|c|c|}
\hline \multirow{2}{*}{ Grupo dos alimentos } & \multicolumn{2}{|c|}{ Dia da semana } & \multicolumn{2}{|c|}{ Final de semana } & \multirow{2}{*}{ p-valor } \\
\hline & $M$ & $\pm \mathrm{DP}$ & $M$ & $\pm \mathrm{DP}$ & \\
\hline Açúcar & 3,1 & $\pm 1,2$ & 3,3 & $\pm 2,5$ & $p>0,05$ \\
\hline Gordura & 1,0 & $\pm 0,7$ & 0,7 & $\pm 0,6$ & $p>0,05$ \\
\hline Leite & 2,1 & $\pm 0,8$ & 1,8 & $\pm 1,0$ & $p>0,05$ \\
\hline Carne & 2,2 & $\pm 0,7$ & 1,6 & $\pm 0,7$ & $p<0,05$ \\
\hline Leguminosas & 3,4 & $\pm 1,2$ & 1,6 & $\pm 1,0$ & $p<0,05$ \\
\hline Frutas & 1,2 & $\pm 0,5$ & 0,4 & $\pm 0,6$ & $p<0,05$ \\
\hline Legumes & 2,2 & $\pm 0,6$ & 0,5 & $\pm 0,5$ & $p<0,001$ \\
\hline Cereais & 4,2 & $\pm 1,4$ & 4,8 & $\pm 2,7$ & $p>0,05$ \\
\hline
\end{tabular}

Obs: população do estudo $=20$ crianças 
representativa do município do Rio de Janeiro, sendo observado um aumento de quase três vezes no consumo de refrigerantes nessa população, quando comparado com os resultados obtidos no Estudo Nacional de Despesa Familiar ${ }^{20}$. O aumento do consumo de refrescos industrializados, refrigerantes, balas e açúcar de adição está associado a uma maior prevalência de obesidade infantil, sendo ainda investigada sua relação com algumas doenças, como a cárie dental e as doenças cardiovasculares ${ }^{23}$.

Foi observada uma diferença significativa entre as porções consumidas durante a semana no grupo de legumes e frutas (2,2 e 1,2 porções respectivamente) e o consumo do final de semana (0,5 e 0,4 porção, respectivamente) (Tabela 2), pois na creche são oferecidos diariamente sucos, sobremesa e outras preparações com alimentos desses grupos. Apesar de o consumo de legumes e frutas ser maior durante a semana, esse não atingiu o porcionamento recomendado por Philippi et al. $^{10}$ ( 3 e 2 porções de legumes e frutas). Resultados encontrados no estudo nacional dos Estados Unidos mostraram que crianças consomem menos frutas e legumes e mais gordura e energia do que o recomendado para a idade ${ }^{24}$.

Encontrou-se uma diferença significativa no grupo de carne: seu consumo durante a semana (2,2 porções) foi maior que no final de semana (1,6 porções). O consumo de carnes durante a semana foi um pouco maior do que o recomendado pela pirâmide brasileira (2 porções). Esse resultado difere do encontrado por Harwood et al. ${ }^{25}$, que, ao estudarem pré-escolares mexicanos-americanos de baixa renda $(n=211)$, verificaram que o grupo das carnes encontrava-se abaixo da metade da porção recomendada ${ }^{25}$. Já o consumo de leguminosas durante a semana foi significativamente maior do que no final de semana, porém, tanto durante a semana como no final de semana, o seu consumo atingiu o recomendado por Philippi et al. ${ }^{10}$ (1 porção).

O número de porções do grupo do leite não atingiu a recomendação (3 porções) da pirâmide brasileira tanto durante a semana $(2,2$ porções) como no final de semana (1,9 porções). No estudo realizado por Schwartz ${ }^{19}$, encontrou-se uma diminuição do consumo de leite nas crianças que consumiam excesso de bebidas industrializadas, levando a um aporte inadequado de cálcio e vitamina $D$. A ingestão inadequada desse grupo impede o crescimento e a mineralização do osso, contribuindo para o maior risco de osteoporose no indivíduo adulto ${ }^{26}$.

O grupo dos cereais também não atingiu a recomendação da pirâmide infantil brasileira (5 porções) (Tabela 2). No final de semana o consumo foi de 4,8 porções e durante a semana 4,2 porções, principalmente pelo consumo de biscoitos salgadinhos e recheados incluídos nesse grupo. Briley et al. ${ }^{27}$, ao avaliarem os cardápios de creches no Texas, observaram que o consumo de cereais pelas crianças era metade da porção recomendada pela pirâmide americana e $20 \%$ da população estudada não consumiam alimentos integrais. Os cereais e alimentos integrais não estão presentes com freqüência nos cardápios das creches, porém alguns autores consideram-nos de grande importância, pois podem aumentar o aporte nutricional nas crianças da creche por serem fortificados com vitaminas e minerais ${ }^{23}$.

Resultados similares com relação à inadequação do consumo do grupo dos legumes, frutas, carnes, leite e cereais são encontrados em outros estudos. Na pesquisa realizada por Cunha ${ }^{28}$, em Uberaba, SP, com pré-escolares, encontrou-se a seguinte freqüência de consumo de alimentos expresso em número de porções ingeridas por dia: legumes (1,36 porções), frutas (2,04 porções), carnes (1,79 porções), leite (2,57 porções), cereais $\left(3,16\right.$ porções) e açúcar (1,51 porções) ${ }^{28}$. Houve uma inadequação da dieta principalmente no grupo dos legumes, frutas, carnes, leite e cereais. Munoz et al. ${ }^{29}$ relataram achados similares em uma amostra nacional de crianças e adolescentes na qual 30\% não atingiram as porções recomendadas do grupo das frutas, grãos, carne e leite e $36 \%$ para o grupo dos vegetais ${ }^{29}$.

São poucos os estudos sobre o consumo alimentar de crianças brasileiras. Foram verificadas 
mudanças no hábito alimentar das famílias na última Pesquisa Nacional sobre Orçamento Familiar (POF) ${ }^{30}$. Nessa pesquisa observou-se a intensificação do consumo relativo de carnes em todas as áreas metropolitanas do país. O consumo relativo de leite e derivados prosseguiu aumentando, apesar da estagnação e redução do consumo de leguminosas, cereais, tubérculos, verduras, legumes e frutas e sucos naturais e da ascensão do consumo de açúcar refinado e refrigerantes. Em linhas gerais, esses foram os aspectos mais marcantes e negativos da evolução do padrão alimentar observado entre as POF 1988 e $1996^{30}$.

\section{O N CLUS Ã O}

A fase pré-escolar é um excelente momento para a formação de hábitos alimentares saudáveis que respeitem as características de cada criança. O estabelecimento de guias alimentares tem a finalidade de orientar a população quanto à seleção, à forma e à quantidade de alimentos a serem consumidos.

Neste estudo observou-se que, no final de semana, é maior a inadequação da dieta das crianças quando comparada com a oferecida na creche durante a semana. Há necessidade de um maior cuidado em relação à alimentação desse grupo, principalmente no final de semana, pois nessa faixa etária ocorre a incorporação de novos hábitos alimentares implicando o conhecimento de novos sabores, texturas e cores, experiências sensoriais que influenciarão diretamente o padrão alimentar a ser adotado pela criança em toda a sua vida.

Faz-se necessário orientar os responsáveis quanto às inadequações alimentares para que ocorra uma correta complementação na residência, com objetivo de melhorar a qualidade da dieta. A avaliação do consumo alimentar de crianças por meio da pirâmide alimentar desenvolvida por Philippi et al. ${ }^{10}$ poderá servir para pais, educadores e profissionais de saúde como um guia prático de orientação da alimentação de crianças.
REFERÊ NCIAS

1. Harris S, Black R, Harvey AG. Dietary guidelines: past experience and new approaches. J Am Diet Assoc. 2003; 103(Suppl 2):S3-S4.

2. World Health Organization. Preparation and use of food-based dietary guidelines. Nutrition Programme. Geneva: WHO; 1996.

3. Davis CA, Britten P, Myers EF. Past, present, and future of the Food Guide Pyramid. J Am Diet Assoc. 2001; 101(8):881-5.

4. Slavin JL. Implementation of dietary modifications. Am J Med. 1999; 106(1A):46S-9S; discussion 50S-1S.

5. United States Departament of Agriculture. United States Departament of Health and Human Services. The Food Guide Pyramid. Washington (DC): US Government Printing Office; 1992. Home and Garden Bulletin $n^{\circ} 252$.

6. Brady LM, Lindquist CH, Herd SL, Goran MI. Comparison of children's dietary intake patterns with US dietary guidelines. Br J Nutr. 2000; 84(3):361-7.

7. Welsh S, Davis C, Shaw A. Development of the food guide pyramid. Nutr Today. 1992; 27(26):12-23.

8. Davis CA, Escobar A, Marcoe KL, Tarone, C, Shaw $A$, Saltos $S$, et al. Food guide pyramid for young children 2 to 6 years old: Technical Report on Background and Development. Washington (DC): US Department Agriculture, Center for Nutrition Policy and Promotion; 1999. CNPP-10.

9. Philippi ST, Cruz ATR, Ferreira MN, Coutinho RLR. Alimentação saudável na infância e na adolescência. In: Curso de atualização em alimentação e nutrição para professores da rede pública de ensino. Piracicaba: Escola Superior de Agricultura Luiz de Queiroz; 2000. p.46-60.

10. Philippi ST, Cruz ATR, Colucci ACA. Pirâmide alimentar para crianças de 2 e 3 anos. Rev Nutr. 2003; 16(1):5-19.

11. Rodrigo CPS, Bartrina JA. Diário o registro dietético: métodos de doble pesada. In: Majem LS, Bartrina JA, Verdú JM. Nutricion y Salud Pública: métodos, bases científicas y aplicaciones. Barcelona: Masson; 1995. p.107-95.

12. Aquino RC, Philippi ST. Consumo infantil de alimentos industrializados e renda familiar na cidade de São Paulo. Rev Saúde Pública. 2002; 36(6):655-60.

13. Szarfarc SC, Monteiro CA, Meyer M, Tudisco ES, Reis IM. Estudo das condições de saúde das crianças do Município de São Paulo 1984/1985: 
Consumo Alimentar. Rev Saúde Pública. 1988; 22(4):266-72.

14. Magaretts BM, Nelson M. Design concepts in nutritional epidemiology. New York: Oxford University Press; 1997.

15. Flores N. Metodologia en encuestas alimentarias entre pré-escolares. Arch Latinoam Nutr. 1972; 33(6):359-84.

16. Basiotis PP, Welsh SO, Cronin FJ, Kelsay JL, Mertz $W$. Number of days of food intake records required to estimate individual and group nutrient intakes with defined confidence. J Nutr. 1987; 117(9):1638-41.

17. Menchú MT. Revision de las metodologias para estudios del consumo de alimentos. Guatemala: OPS/INCAP; 1993. INCAP - Publicación ME/15.

18. Rampersaud GC, Bailey LB, Kauwell GP. National survey beverage consumption data for children and adolescents indicate the need to encourage a shift toward more nutritive beverages. J Am Diet Assoc. 2003; 103(1):97-100.

19. Schwartz RP. Soft drinks taste good, but the calories count. J Pediatr. 2003; 142(6):599-601.

20. Associação Brasileira das Indústrias da Alimentação [ABIA]. Consumo de alimentos em novo patamar. São Paulo; 1997. ABIA Informa, 272.

21. Galeazzi MAN, Domene SMA, Sichieri R. Estudo multicêntrico sobre consumo alimentar. Cad Debate. 1997; ( $n^{\circ}$ especial):1-62.

22. Sichieri R. Epidemiologia da obesidade. Rio de Janeiro: UERJ; 1998.

23. Kranz S, Siega-Riz AM. Sociodemographic determinants of added sugar intake in preschoolers 2 to 5 years old. J Pediatr. 2002; 140(6):667-72.

24. Krebs-Smith SM, Cook A, Subar AF, Cleveland L, Friday J, Kahle LL. Fruit and vegetable intakes of children and adolescents in the United States. Arch Pediatr Adolesc Med. 1996; 150(1):81-6.

25. Kaiser LL, Melgar-Quinonez HR, Lamp CL, Johns MC, Sutherlin JM, Harvood JO. Food security and nutritional outcomes of preschool-age MexicanAmerican children. J Am Diet Assoc. 2002; 102(7):924-9.

26. Nicklas T, Johnson R. American Dietetic Association. Position of the American Dietetic Association: Dietary guidance for healthy children ages 2 to 11 years. J Am Diet Assoc. 2004; 104(4):66077. Erratum in: J Am Diet Assoc. 2004; 104(7):1075.

27. Briley ME, Jastrow S, Vickers J, Roberts-Gray C. Can ready-to-eat cereal solve common nutritional problems in child-care menus? J Am Diet Assoc. 1999; 99(3):341-3.

28. Cunha SFC. Guia alimentar da pirâmide: experiência em Uberaba, MG. In: Workshop Instituto Danone. Alimentação Equilibrada para População Brasileira. Florianópolis; 1998.

29. Munoz KA, Krebs-Smith SM, Ballard-Barbash R, Cleveland LE. Food intakes of US children and adolescents compared with recommendations. Pediatrics. 1997; 100(3 Pt 1):323-9. Erratum in: Pediatrics. 1998; 101(5):952-3.

30. Monteiro CA, Mondini L, Costa RL. Mudanças na composição e adequação nutricional da dieta familiar nas áreas metropolitanas do Brasil entre 1988 e 1996. In: Monteiro, CA. Velhos e novos males da saúde no Brasil: a evolução do país e de suas doenças. São Paulo: Hucitec; 2000. p.359-74.

Recebido para publicação em 21 de maio e aceito em 18 de outubro de 2004. 